\title{
Comprensión actual de los biomarcadores circulantes en la hipertensión pulmonar debida a enfermedad de ventrículo izquierdo
}

\author{
Noah Todd ${ }^{\mathrm{a}}$ Yen-Chun Lai ${ }^{\mathrm{a}, \mathrm{b}}$ \\ a'División de Medicina Pulmonar, Cuidados Críticos, del Sueño y Ocupacional, Departamento de Medicina, Escuela de Medicina \\ de la Universidad de Indiana, Indianápolis, IN, Estados Unidos; ' ${ }^{b}$ Departamento de Anatomía, Biología Celular y Fisiología, Escuela de \\ Medicina de la Universidad de Indiana, Indianápolis, IN, Estados Unidos
}

\author{
Palabras clave \\ Hipertensión pulmonar · HP grupo 2 · HP-ICFEp . \\ ICFEp - insuficiencia cardiaca con fracción de eyección \\ preservada Biomarcadores
}

\section{Resumen}

La hipertensión pulmonar debida a enfermedad del ventrículo izquierdo (HP-EVI, grupo 2), especialmente en el contexto de insuficiencia cardiaca con fracción de eyección preservada (ICFEp), es la causa más frecuente de HP. A pesar de su prevalencia, por el momento no disponemos de terapias efectivas para la HP-EVI. Esto se debe en gran medida a la falta de una definición concisa de los fenotipos hemodinámicos, la existencia de huecos significativos en nuestra comprensión de la patología subyacente y del impacto de comorbilidades asociadas, así como a la ausencia de biomarcadores específicos que pudieran ayudar en el diagnóstico temprano y el manejo de este complejo síndrome. Los lineamientos actuales recomiendan emplear el péptido natriurético tipo B (BNP) y el proB-
NP N-terminal (ProBNP-NT) como biomarcadores para el diagnóstico y el pronóstico de la insuficiencia cardiaca (IC) y la HP. Recientemente, también se identificó a la endotelina 1 (ET-1), el factor de crecimiento endotelial vascular D (VEGF-D) y el microRNA-206 como potenciales biomarcadores circulantes para pacientes con HP-EVI. El objetivo de esta revisión es presentar el estado actual de nuestro conocimiento sobre los biomarcadores circulantes que pueden emplearse para guiar las futuras investigaciones hacia el diagnóstico, refinar el fenotipo específico de los pacientes y desarrollar estrategias terapéuticas para la HP-EVI, con atención particular en la HP-ICFEp. Asimismo, se resumen los biomarcadores identificados en modelos preclínicos de HP-EVI.

\section{Introducción}

La hipertensión pulmonar debida a enfermedad del ventrículo izquierdo (HP-EVI, grupo 2) es la causa más común de HP y es un creciente problema de salud pública, con alta mortalidad y morbilidad [1-3]. De hecho, la presencia de HP en pacientes con EVI se ha asociado con índices de mortalidad hasta 5.6 veces mayores con respecto a pacientes sin HP [4]. La disfunción sistólica y diastólica del ventrículo izquierdo (VI), la enfermedad valvular del lado izquierdo (enfermedad de la válvula aórtica y mitral) y la desregulación metabólica son factores coadyuvantes que condu- cen a una mayor presión de llenado en el VI y al subsecuente desarrollo de HP. Entre ellas, la HP atribuida a la disfunción diastólica del VI, también conocida como HP asociada con insuficiencia cardiaca con fracción de eyección preservada (HP-ICFEp), es la forma más común, aunque los reportes de prevalencia varían de 23 a $83 \%$ debido a diferencias en las definiciones y los métodos diagnósticos usados hasta la fecha [5-7]. En adición a la ambigüedad ya existente en la definición de este complejo síndrome, el $6^{\circ}$ Simposio Mundial sobre HP (Niza, 2018) redefinió recientemente la HP-EVI, que ahora consiste en una presión arterial pulmonar media (mPAP) mayor de $20 \mathrm{~mm} \mathrm{Hg}$ (anteriormente era al menos (c) 2021 The Author(s)

Published by S. Karger GmbH, Freiburg

This article is licensed under the Creative Commons Attribution 4.0 International License (CC BY) (http://www.karger.com/Services/Open AccessLicense). Usage, derivative works and distribution are permitted provided that proper credit is given to the author and the original publisher. 


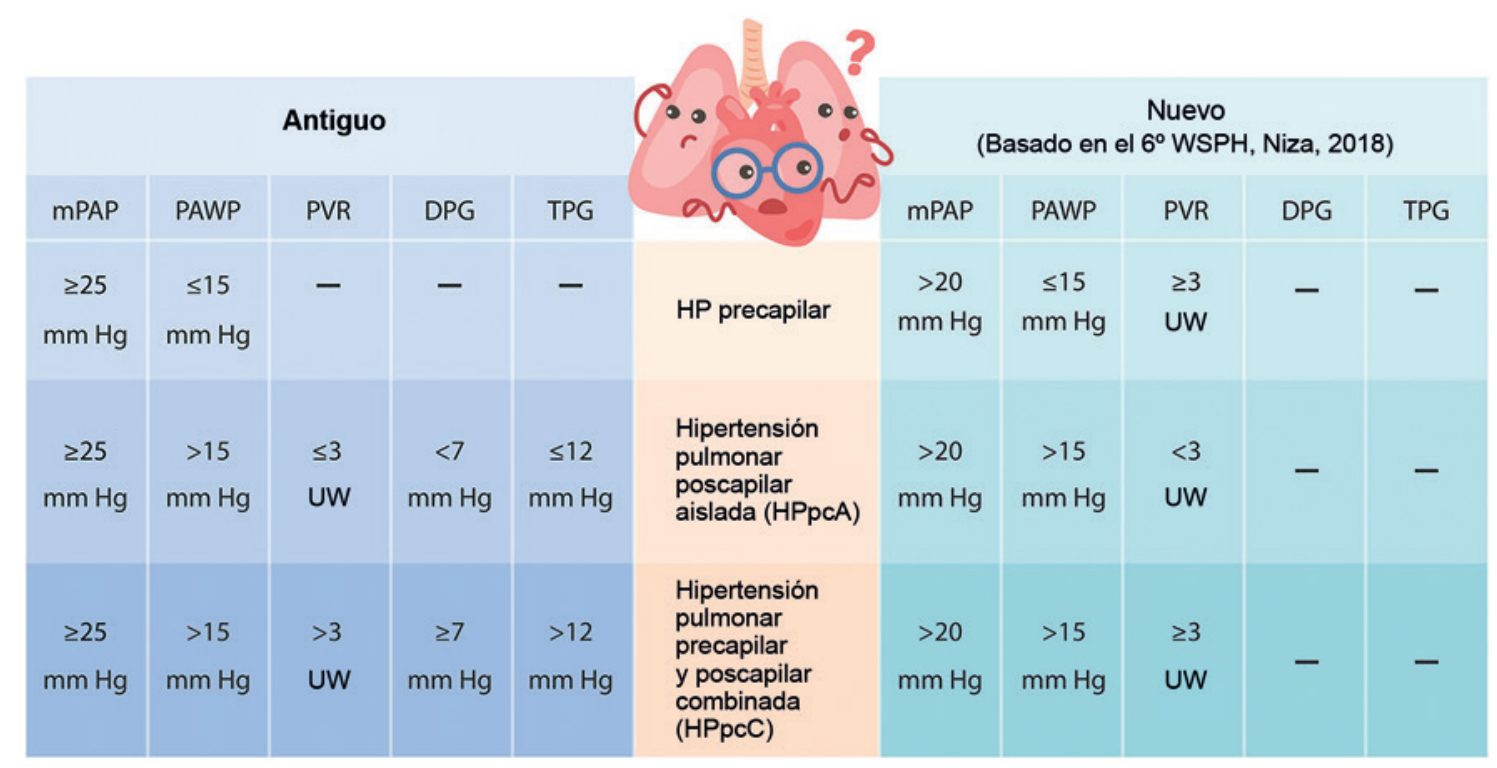

Fig. 1. Definición hemodinámica actualizada y clasificación clínica de hipertensión pulmonar (con base en el 60 Simposio Mundial sobre HP, Niza, 2018). mPAP, presión arterial pulmonar media; PAWP, presión arterial pulmonar de enclavamiento; TPG, gradiente transpulmonar (definido como mPAP - PAWP); PVR, resistencia vascular pulmonar (definida como TPG/gasto cardiaco); DPG, gradiente de presión diastólica (definido como PAP diastólica - PAWP).

$25 \mathrm{~mm} \mathrm{Hg}$ ) y una presión arterial pulmonar de enclavamiento (PAWP) mayor de $15 \mathrm{~mm} \mathrm{Hg}$ (Figura 1) [8-11]. Aunque este cambio refleja los reportes recientes que describen un mayor riesgo de progreso de la enfermedad en pacientes con una elevación leve en la mPAP (21 a $24 \mathrm{~mm} \mathrm{Hg}$ ), este cambio podría causar mayor confusión entre los practicantes clínicos cuando traten de diagnosticar la HP en pacientes $[9,12]$.

Tanto las presiones de llenado del VI anormalmente elevadas en la ICFEp como la insuficiencia cardiaca con fracción de eyección reducida (ICFEr, insuficiencia cardiaca sistólica) y la enfermedad valvular pueden conducir a un aumento en la presión en la aurícula izquierda (AI), un aumento de volumen en la AI y una menor compliancia en la AI $[5,13]$. Entre los mecanismos celulares básicos que afectan la remodelación del VI y la AI se incluyen la hipertrofia de miocitos, la sobreexpresión del péptido natriurético tipo B miocárdico o cerebral en el miocardio del ventrículo o la aurícula con insuficiencia (en respuesta a los incrementos de presión o a la sobrecarga de volumen), la disfunción endotelial, el estrés oxidativo vascular, la inflamación, la fibrosis intersticial y varias anormalidades metabólicas [14-18]. Una mayor presión en la AI puede verse reflejada en la circulación pulmonar, incrementando la presión venosa pulmonar, que a su vez se transfiere a los capilares pulmonares, causando daño a la barrera alveolar-capilar (fenómeno también conocido como falla por estrés alveolar-capilar). Conforme la enfermedad progresa, los cambios estructurales y funcionales regulados por la elevación crónica en la presión capilar pueden provocar vasoconstricción pulmonar, reducir la biodisponibilidad del óxido nítrico (NO), incrementar la producción de endotelina 1 (ET-1) y promover la remodelación de las arterias y venas pulmonares, con varias combinaciones de proliferación de la íntima, hipertrofia de la media y engrosamiento de la adventicia $[13,17,19-$
22]. Estas anormalidades en la vasculatura pulmonar pueden incrementar aún más la mPAP y la PAWP, dando como resultado un amento en la poscarga del ventrículo derecho (VD) y en última instancia causando insuficiencia cardiaca derecha. Dependiendo de la extensión de las anormalidades progresivas en la vasculatura pulmonar (también conocidas como componente precapilar) con respecto a la EVI subyacente, la transmisión retrógrada de la presión de llenado elevada puede causar un aumento en la presión arterial pulmonar de manera proporcional (razón 1:1) o desproporcional (razón >1:1) [17, 23-25]. Con frecuencia, los pacientes sin vasoconstricción pulmonar significativa ni vasculopatía pulmonar intrínseca exhiben una HP proporcional (HP poscapilar aislada, HPpcA). Por otro lado, si la presión de llenado del VI crónicamente elevada desencadena vasoconstricción pulmonar y remodelación precapilar patológica hasta el punto de provocar un gradiente transpulmonar elevado (TPG, definido como mPAP - PAWP mayor de $12 \mathrm{~mm} \mathrm{Hg}$ ), una alta resistencia vascular pulmonar (PVR, definida como TPG/ gasto cardiaco mayor que 3 unidades de Wood), o un gradiente pulmonar diastólico elevado (DPG, definido como PAP diastólica - PAWP mayor o igual a $7 \mathrm{~mm} \mathrm{Hg}$ ), los pacientes exhiben una HP fuera de proporción (HP precapilar y poscapilar combinada, $\mathrm{HPpcC}$ ). La gravedad de la afectación precapilar puede establecerse al medir la TPG, la PVR, y/o la DPG durante la cateterización cardiaca derecha. En el pasado, una TPG $\leq 12 \mathrm{~mm}$ $\mathrm{Hg}$, una PVR $\leq 3$ unidades de Wood y/o una DPG $<7 \mathrm{~mm} \mathrm{Hg}$ sugerían HPpcA, y cuando estos parámetros estaban elevados (TPG >12 mm Hg, PVR >3 unidades de Wood y/o DPG $\geq 7 \mathrm{~mm}$ $\mathrm{Hg}$ ), sugerían HPpcC (8). Estas características se han asociado con mayor mortalidad y hospitalizaciones por padecimientos cardiacos en pacientes con HPpcC asociada con ICFEp (HPpcC-ICFEp) [26, 27]. Sin embargo, la nueva definición a par- 
tir del $6^{\circ}$ Simposio Mundial sobre HP solamente incluye una PVR menor que 3 unidades de Wood para la HPpcA y una PVR de al menos 3 unidades de Wood para la HPpcC (Figura 1) [911 .

A pesar de su significativa prevalencia, junto con una alta morbilidad y mortalidad, hasta el momento no existe tratamiento aprobado por la Administración de Fármacos y Alimentos (FDA) para la HP-EVI. Mientras que se ha demostrado que el uso de vasodilatadores pulmonares es efectivo en el tratamiento de hipertensión arterial pulmonar (HAP, grupo 1), el empleo de estos agentes en pacientes con HP-EVI ha resultado ineficaz o incluso dañino (véase en Fernandez et al. y Vachiéry et al. revisiones recientes y detalladas) $[22,28]$. Esto se debe en parte a la falta de una definición concisa y uniforme para el fenotipo hemodinámico, un escaso entendimiento de la patología subyacente y del impacto de las comorbilidades asociadas, así como a la ausencia de biomarcadores específicos que puedan facilitar el diagnóstico temprano y el manejo de esta heterogénea enfermedad. Los lineamientos actuales recomiendan emplear el BNP y el proBNP N-terminal (ProBNP-NT) como biomarcadores para el diagnóstico y el pronóstico de la IC y la HP [29-31]. Sin embargo, éstos no son específicos para la HP-EVI y varían significativamente con la edad, el sexo, el índice de masa corporal (IMC) y la función renal [30, 31]. Asimismo, se ha comprobado que varios biomarcadores se complementan con los péptidos natriuréticos para guiar el manejo de la enfermedad y poseen valor diagnóstico para distinguir entre $\mathrm{HPpcA}$ y HPpcC. El objetivo de esta revisión es ofrecer un panorama actualizado del estado del conocimiento sobre biomarcadores circulantes que pueden emplearse para guiar las investigaciones futuras hacia el diagnóstico, el refinamiento de fenotipos específicos de los pacientes y el desarrollo de estrategias terapéuticas para la HP-EVI, con atención particular a la HP-ICFEp. Nos centraremos primero en los péptidos natriuréticos, cuyo empleo está bien establecido, y luego revisaremos biomarcadores conocidos que se han propuesto para la HAP y sus nuevas funciones en la HP-EVI. Finalmente, señalaremos algunos de los biomarcadores circulantes emergentes, incluyendo aquellos identificados recientemente en modelos preclínicos de HP-EVI.

\section{Biomarcadores bien establecidos: BNP y ProBNP-NT}

Conforme progresa la disfunción del VI, el estrés diastólico en la pared es el estímulo primario para la expresión de BNP miocárdico, que tiene un papel importante en la regulación de la remodelación cardiaca, la presión arterial y el volumen intravascular [14-16]. El BNP se transcribe y produce principalmente en los cardiomiocitos de los ventrículos como la prohormona proBNP, que posteriormente se escinde para formar el BNP (activo biológicamente, con una vida media de $20 \mathrm{~min}$ ) y su fragmento N-terminal ProBNP-NT (biológicamente inactivo, con una vida media de 70 min). El precursor, el BNP y el ProBNP-NT se secretan directamente en la circulación en respuesta al aumento en la distensión miocárdica mediada por la presión o por sobrecarga de volumen [14-16].

\section{BNP/ProBNP-NT en ICFEp e ICFEr}

Los niveles plasmáticos de BNP y ProBNP-NT están elevados en pacientes con IC y se incrementan en proporción con el grado de disfunción en el VI y la gravedad de los síntomas de ICFEp, ICFEr y enfermedad valvular $[15,32,33]$. De hecho, BNP y ProBNP-NT se encuentran entre los primeros biomarcadores circulantes incluidos en los lineamientos actuales para el diagnóstico y la estratificación de riesgo de IC $[8,31]$. Mientras que los puntos de corte para el BNP y el ProBNP-NT no están bien establecidos para la ICFEp o la ICFEr (véase en Ponikowski et al. y Pieske et al. los valores recomendados más recientes) $[18,30]$, y varios estudios han empleado diferentes umbrales, generalmente se reconoce que sus niveles son, en promedio, más bajos en la ICFEp que en la ICFEr $[30,33]$. Esto puede deberse a la alta prevalencia de obesidad en las poblaciones con ICFEp, puesto que se han reportado niveles desproporcionadamente bajos de BNP y ProBNP-NT en pacientes obesos, lo que puede estar relacionado con los mecanismos que involucran la degradación del péptido natriurético en el tejido adiposo, la resistencia a la insulina y una mayor resistencia pericárdica [32, 34-37]. Los adipocitos expresan fuertemente los receptores de péptido natriurético NPR-A y NPR-C. Puesto que BNP se une tanto a NPR-A y NPR-C, se ha sugerido que un incremento en el tejido adiposo puede llevar a una eliminación más eficiente de BNP en pacientes obesos. Sin embargo, los menores niveles plasmáticos de ProBNP-NT observados en pacientes con IC relacionada con la obesidad no pueden explicarse por el mismo mecanismo, porque el ProBNP-NT no se une a NPR-A ni a NPR-C $[38,39]$. Adicionalmente, Obokata et al. encontraron que en pacientes con ICFEp vinculada con la obesidad es más frecuente la remodelación concéntrica del VI, y muestran un mayor grosor de la grasa en el epicardio, una PAWP más alta, mayor dilatación y disfunción del VD, presión elevada en la aurícula derecha (AD) y un incremento mayor en el volumen total del corazón, junto con niveles menores de ProBNP-NT con respecto a pacientes no-obesos con ICFEp [32]. En ese estudio, la PAWP fue más alta para cualquier nivel de ProBNP-NT en pacientes con ICFEp vinculada con la obesidad que en pacientes no-obesos con ICFEp; sin embargo, la relación entre el ProBNP-NT y la presión transmural en el VI, definida como la presión intracavitaria (PAWP) menos la presión externa aplicada al VI desde el pericardio y el lado derecho del corazón (presión de la AD), no cambió en pacientes con ICFEp, fueran obesos o no [32]. Un incremento en la grasa epicárdica y un mayor volumen cardiaco exacerban la resistencia pericárdica, que puede reducir el esfuerzo en las paredes conforme la presión externa aplicada en el ventrículo aumenta; estos datos podrían sugerir un mecanismo alternativo para la menor concentración de ProBNP-NT observada en pacientes con ICFEp vinculada con la obesidad [32, 37]. Sin embargo, se ha encontrado que incluso elevaciones leves de ProBNP-NT se correlacionan con un mayor riesgo de IC en individuos con obesidad [40].

\section{BNP/ProBNP-NT en HP-ICFEp y HP-ICFEr}

Empleando un punto de corte de $>100$ pg/ml, en un análisis del Programa de ICFEp de la Northwestern University se reportó que
94

Kompass Neumol 2021;3:92-100 DOI: $10.1159 / 000519806$ 
Tabla 1. Estudios recopilados sobre BNP/ProBNP-NT en HP-EVI

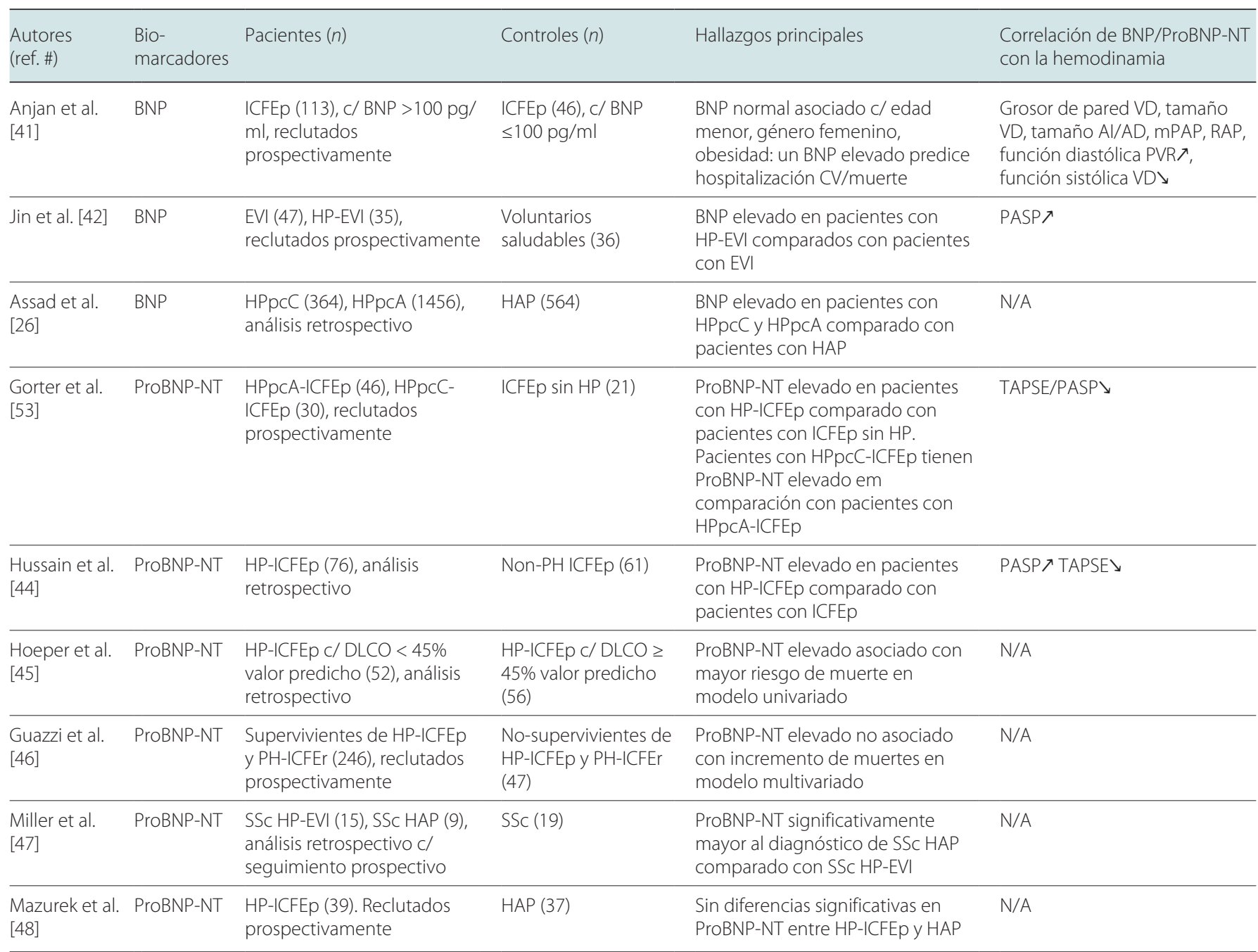

BNP, péptido natriurético tipo B; HPpcC, hipertensión pulmonar precapilar y poscapilar combinada; CV, cardiovascular; DLCO, capacidad de difusión del pulmón para el monóxido de carbono; ICFEp, insuficiencia cardiaca con fracción de eyección preservada; HPpcA, hipertensión pulmonar poscapilar aislada; Al, aurícula izquierda; LAD, diámetro auricular izquierdo; EVI, enfermedad ventricular izquierda; LVEDP, presión de fin de diástole ventricular izquierda; LVEF, fracción de eyección ventricular izquierda; mPAP, presión arterial pulmonar media; ProBNP-NT, fragmento N-terminal del propéptido natriurético tipo B; HAP, hipertensión arterial pulmonar; PASP, presión sistólica en la arteria pulmonar; PH, hipertensión pulmonar; HP-EVI, hipertensión pulmonar asociada con enfermedad del ventrículo izquierdo; PVR, resistencia vascular pulmonar; AD, aurícula derecha; RAP, presión auricular derecha; VD, ventrículo derecho; RVSP, presión sistólica ventricular derecha; SSc, esclerosis sistémica; TAPSE, excursión sistólica del anillo tricúspide; $\nearrow$ indica una correlación positiva con el biomarcador correspondiente; \ indica una correlación inversa con el biomarcador correspondiente.

hasta $70 \%$ de los pacientes con ICFEp confirmada tienen niveles elevados de BNP, que se asocian con valores significativamente más altos de mPAP, presión en la AD y PVR, junto con tasas más altas de hospitalización por IC, hospitalización por razones cardiovasculares y muerte, en comparación con pacientes con ICFEp y niveles «normales» de BNP $(\leq 100$ pg/ml) [41]. Se encontró que los niveles elevados de BNP también se asociaron con un incremento en la presión sistólica en la arteria pulmonar (PASP) en pacientes con HP vinculada con ICFEp, ICFEr o enfermedad valvular [42]. Se observaron niveles significativamente más altos de BNP en pacientes con $\mathrm{HPpcC}$ (50\% de los cuales tenían una fracción de eyección en el VI <50\%) con respecto a los de pacientes con HAP [25]. De manera similar, se encontró que pacientes con
HPpcC-ICFEp tenían niveles aproximadamente 1.8 veces más altos de ProBNP-NT que los pacientes con IpcHP-ICFEp; este hallazgo se asoció con una mayor frecuencia de hospitalizaciones por IC, un menor acoplamiento VD-vascular (determinado por el cociente entre la excursión sistólica del anillo tricúspide y el valor de PASP, TAPSE/PASP), alteraciones en la función del VD y una capacidad para ejercitarse gravemente deprimida [43]. Hussain et al. reportaron que los valores globales de ProBNP-NT fueron mayores en pacientes con ICFEp y con HP, en comparación con pacientes sin HP. Los niveles de ProBNP-NT también fueron mayores en pacientes con ICFEp con disfunción en el VD (TAPSE disminuida) a pesar de la ausencia de HP. En este estudio de cohorte se encontró una correlación negativa moderada entre los niveles 
de ProBNP-NT y el cociente TAPSE/PASP [44]. Un análisis retrospectivo que Hoeper et al. realizaron en 108 pacientes diagnosticados con HP-ICFEp reveló que cada incremento de $100 \mathrm{ng} / \mathrm{l}$ en los niveles de ProBNP-NT se asocia con un mayor riesgo de muerte [45]. Guazzi et al. también demostraron que los valores de ProBNP-NT fueron mayores entre pacientes con ICFEp y HP-ICFEr que no sobrevivieron, en comparación con los supervivientes, aunque esta diferencia no se consideró significativa en el análisis multivariado de la supervivencia [46]. Algunos hallazgos recientes sobre BNP/ProBNP-NT en HP-EVI se resumen en la Tabla 1. En contraste, Miller et al. reportaron que los niveles de ProBNP-NT fueron menores en pacientes con esclerosis sistémica con HP temprana debida a EVI que cuando se debió a HAP [47]. Mazurek et al. mostraron que los niveles de ProBNP-NT fueron similares en pacientes con HP-ICFEp y con HAP [48]. La razón de estas discrepancias no queda clara de inmediato. Mientras los niveles de BNP y ProBNP-NT no se han comparado dentro de todas las poblaciones de pacientes con HP y no puede emplearse aún para diferenciar entre HP precapilar y HP-EVI, el incremento en los niveles circulantes de estos biomarcadores parece correlacionarse bien con el empeoramiento en el desenlace clínico, particularmente en pacientes con HP-ICFEp. La cuestión de si el monitoreo de BNP o de ProBNP-NT es significativo y efectivo para identificar a pacientes con alto riesgo o para guiar la terapia específica y mejorar el resultado en pacientes con HP-EVI requiere investigarse más a fondo.

\section{Biomarcadores conocidos que se asocian con la EVI: ET-1}

Como se mencionó anteriormente, la elevación crónica en la presión capilar pulmonar puede causar una menor compliancia vascular pulmonar, anormalidades estructurales y vasoconstricción en la vasculatura pulmonar. Se cree que la disfunción endotelial, que conduce a una reducción en la producción de $\mathrm{NO}$ y un incremento en los niveles de ET-1, es un factor significativo en estos cambios [13, 17, 19, 21, 22]. ET-1 es el vasoconstrictor endógeno más potente conocido hasta el momento, y con frecuencia se ha reportado que sus niveles están aumentados en pacientes con ICFEp, ICFEr y enfermedad valvular del lado izquierdo [49-53]. La hormona se deriva del péptido prepro-ET-1, el cual se corta proteolíticamente para producir un intermediario de 39 aminoácidos, Big ET-1, seguido de inmediato por la producción del péptido vasoactivo de 21 aminoácidos por parte de las enzimas convertidoras de endotelina (ECEs) (Figura 2) [54]. La producción y liberación de Big ET-1 y ET-1 se ven promovidas en respuesta a un mayor estrés en el miocardio, un esfuerzo cortante, bajos niveles de estrógeno, condiciones hiperglucémicas, colesterol LDL oxidado, niveles elevados de citocinas proinflamatorias y otras condiciones comúnmente involucradas en la progresión de la EVI, aunque Big ET-1 tiene una potencia vasoconstrictora al menos dos órdenes de magnitud menor que la ET-1 madura [55, 56]. La ET-1 actúa en dos diferentes receptores acoplados con la proteína $\mathrm{G}, \mathrm{ET}_{\mathrm{A}}$ y $\mathrm{ET}_{\mathrm{B}}$. Los receptores $\mathrm{ET}_{\mathrm{A}}$ se localizan predominantemente en las células de músculo liso vasculares y los miocitos,

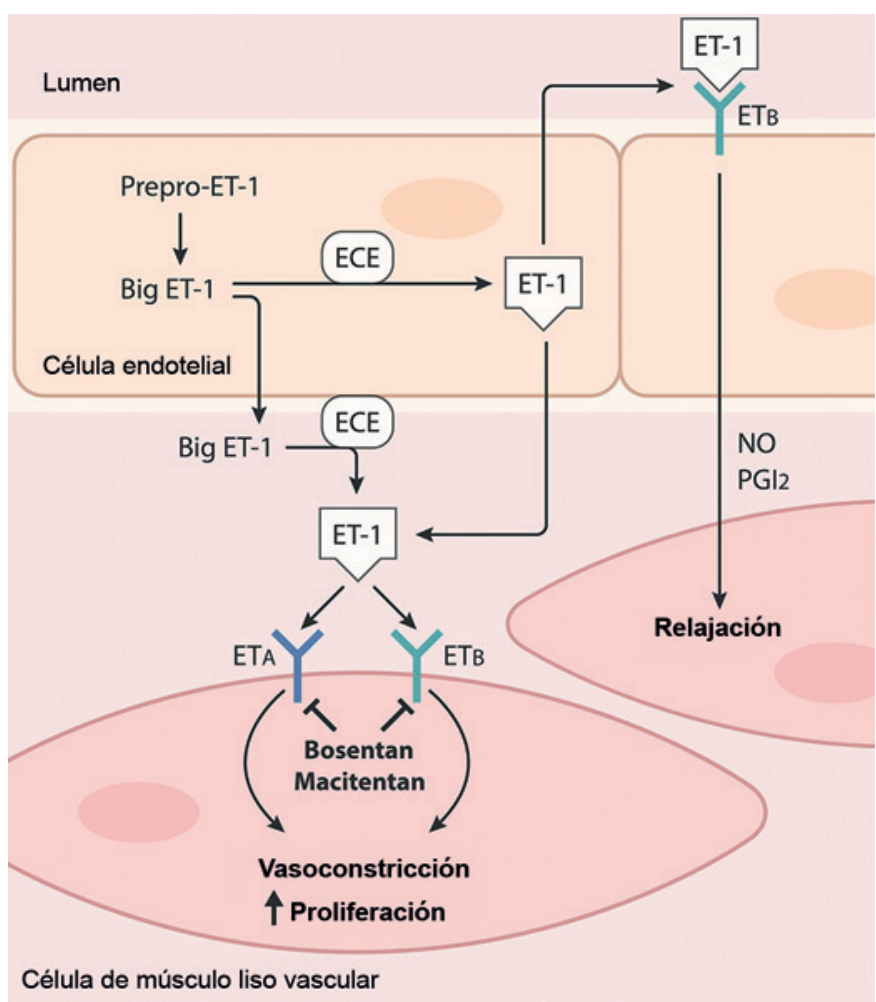

Fig. 2. Producción y liberación de endotelina 1 (ET-1) y acciones mediadas por receptores ET en células de músculo liso vasculares y células endoteliales. La ET-1 se deriva de prepro-ET-1, el cual sufre corte proteolítico para producir un intermediario de 39 aminoácidos, Big ET-1, seguido por la producción del péptido vasoactivo de 21 aminoácidos mediante las enzimas convertidoras de endotelina (ECEs). ET-1 puede activar receptores de endotelina tipo $A\left(E T_{A}\right)$ y tipo $B\left(E T_{B}\right)$. Los receptores $E T_{A}$ se localizan predominantemente en células de músculo liso vasculares, mientras que los receptores $E T_{B}$ residen en las células de músculo liso vasculares y células endoteliales. La activación de $E T_{A}$ o $\mathrm{ET}_{\mathrm{B}}$ en células de músculo liso vasculares produce vasoconstricción y proliferación. La activación de ET induce vasodilatación transitoria en células endoteliales por liberación de óxido nítrico $(\mathrm{NO})$ y prostaciclina $\left(\mathrm{PGI}_{2}\right)$.

y se conocen por sus potentes y duraderas respuestas vasoconstrictoras y proliferativas a la ET-1. La activación de $\mathrm{ET}_{\mathrm{B}}$, por otro lado, produce vasoconstricción en células de músculo liso, pero induce una vasodilatación transitoria en las células endoteliales al liberar NO y prostaciclina. Se encontraron niveles elevados de ET-1 en la sangre de pacientes con HP [51, 52]. Asimismo, se reportaron niveles plasmáticos aumentados de Big ET-1 en pacientes con HAP [57]. Recientemente, Meoli et al. reportaron que los niveles de ET-1 son más elevados en muestras de plasma recolectadas en la posición de enclavamiento de pacientes con $\mathrm{HPpcC}$ ICFEp con respecto a pacientes con ICFEp con HPpcA o sin HP [58]. Aunque el tamaño de la muestra era pequeño, en este estudio se reportó que la concentración plasmática de ET-1 en el enclavamiento se correlacionó fuertemente con el valor de PVR en pacientes con HPpcC-ICFEp. El estudio en una cohorte ligeramente más grande realizado por Chowdhury et al. mostró que, independientemente de su asociación con $\mathrm{HPpcC}$ o HPpcA, la concentración plasmática de ET-1 en el enclavamiento es más alta en pacientes con HP-ICFEp y se asocia con HP, PVR y hospitali-
96

Kompass Neumol 2021;3:92-100 DOI: $10.1159 / 000519806$ 
zación por insuficiencia cardiaca a 1 año, en comparación con pacientes con ICFEp, pero sin HP [59]. Similarmente, Obokata et al. reportaron que los pacientes con diagnóstico confirmado de ICFEp muestran niveles elevados de pro-ET-1 C-terminal (ProET-1-CT), un precursor estable circulante de ET-1, cuya magnitud se asoció con una mayor presión arterial pulmonar y una peor compliancia arterial pulmonar en reposo y durante el ejercicio [52]. En este estudio, el aumento en los niveles plasmáticos de ProET-1-CT también mostró alta exactitud diagnóstica para identificar pacientes con reserva vascular pulmonar anormal durante el ejercicio. Aunque ET-1 podría tener un papel en la fisiopatología de la HP-ICFEp, los resultados del empleo de antagonistas de los receptores de la endotelina aprobados por la FDA para el tratamiento de la HAP han sido más bien decepcionantes en el tratamiento de pacientes con HP-ICFEp. El estudio piloto del bosentan, un antagonista dual de los receptores $\mathrm{ET}_{\mathrm{A}} \mathrm{y}$ $\mathrm{ET}_{\mathrm{B}}$, no mostró signos de beneficio en pacientes con HP-ICFEp [60]. Más aún, este tratamiento podría incluso ser perjudicial en las poblaciones combinadas de pacientes con HPpcC- e HPpcA-ICFEp. El bosentan mostró efectos nocivos similares durante un ensayo clínico para el tratamiento de pacientes con HP-ICFEr [61]. El ensayo MELODY-1, donde a los pacientes con HPpcC ( $>75 \%$ de fracción de eyección normal) se les asignó aleatoriamente un tratamiento de 12 semanas con macitentan, un antagonista dual de los receptores $\mathrm{ET}_{\mathrm{A}}$ y $\mathrm{ET}_{\mathrm{B}}$, o un placebo, mostró una mayor incidencia de efectos colaterales en el grupo con macitentan (principalmente retención de fluidos) sin mejoría significativa en ninguno de los resultados exploratorios [62]. Actualmente, el ensayo SERENADE, un ensayo clínico en fase IIb, está reclutando pacientes con ICFEp asociada con enfermedad vascular pulmonar o disfunción del VD para evaluar los efectos a largo plazo (24-52 semanas) del tratamiento con macitentan (NCT03153111).

\section{Biomarcadores emergentes: VEGF-D/FIGF}

Además de las respuestas vasoconstrictoras y proliferativas mediadas por la ET-1, el aumento en la secreción de citocinas inflamatorias y factores de crecimiento (p. ej., el factor de crecimiento transformante alfa 1, TGF- $\alpha 1$; el factor de crecimiento endotelial vascular, VEGF, y la interleucina 1, IL1) también se han asociado con cambios estructurales y funcionales en respuesta al incremento retrógrado en la presión capilar pulmonar [22]. Entre ellos, el papel del factor de crecimiento endotelial vascular D (VEGF-D) se ha evaluado recientemente en la HP-EVI. VEGF-D es un factor secretado que regula la angiogénesis, la linfangiogénesis y la permeabilidad vascular $[63,64]$. Se sintetiza y secreta como un precursor más grande, que subsecuentemente sufre proceso proteolítico en los extremos N-y C- para producir las formas maduras. El VEGF-D sin procesar es selectivo para el receptor 3 del factor de crecimiento endotelial vascular (VEGFR-3), el cual se expresa principalmente en células endoteliales linfáticas, mientras que el VEGF-D maduro activa a VEGFR-3 y a VEGFR-2; el último tipo de receptores se encuentra tanto en células endoteliales linfáticas como vasculares [64]. Además de modular el crecimiento de vasos sanguíneos y linfáticos, se ha demostrado que VEGF-D induce fibrogénesis cardiaca al estimular el crecimiento y la migración de miofibroblastos, junto con la síntesis de colágeno tipo I [65]. La expresión de VEGF-D se aumenta por acción del blanco mecanístico (anteriormente de mamífero) de la rapamicina (mTOR), un regulador maestro del crecimiento, la proliferación y la supervivencia celular que se ha visto implicado en la HAP, la linfangioleiomiomatosis (LAM) y el cáncer [66]. Aunque la administración por un vector adenovirus de un gen que codifica el VEGF-D maduro ha mejorado la perfusión al miocardio en cerdos y avanzó a los estudios de fase I/IIa en pacientes con angina refractaria [67, 68], se han reportado niveles elevados de VEGF-D circulante en pacientes con IC, fibrilación auricular, choque isquémico, HAP, hipertensión pulmonar tromboembólica crónica (CTEPH) y LAM [69-73]. Se encontraron niveles más elevados de VEGF-D en pacientes con congestión pulmonar [70]. También se encontró que la elevación de los niveles de VEGF-D es un predictor de la mortalidad por cualquier causa en pacientes con enfermedad arterial coronaria (CAD) y permitió diferenciar sujetos con IC de pacientes con disnea aguda $[70,74]$. Recientemente, Säleby et al. reportaron que los niveles de VEGF-D se encontraban elevados en pacientes con HAP, CTEPH, HP-ICFEp y HP-ICFEr en comparación con sujetos control y con pacientes con IC, pero sin HP; los niveles del marcador fueron significativamente más altos en pacientes con ICFEr con respecto a todas las demás etiologías de HP [72]. Cabe señalar que los niveles plasmáticos de VEGF-A fueron mayores en pacientes con HAP, CTEPH y HP-EVI que en los controles, aunque existe controversia sobre la presencia o ausencia de diferencias significativas en los niveles de VEGF-A entre diferentes etiologías de HP [71, 72]. Sin embargo, los datos son más consistentes para VEGF-D, y muestran niveles aumentados en casos de HP-EVI con respecto a pacientes con HAP y CTEPH. Adicionalmente, los niveles de la tirosina quinasa-1 similar a fms, soluble (sFlt-1, también conocida como VEGFR-1 soluble o sVEGFR-1) se encontraron significativamente elevados en pacientes con HP-EVI en comparación con controles, con pacientes con HAP y con pacientes sin HP-EVI [71]. Estos resultados concuerdan con un estudio realizado por Houston et al., en el que los autores mostraron que los niveles aumentados de VEGF-D se correlacionan con una mayor PAWP, menor gasto cardiaco y niveles más altos de BNP en pacientes con insuficiencia cardiaca [75]. También se encontró una fracción de eyección reducida (30 \pm 18 vs. $49 \pm$ $22 \%$ ) en sujetos con niveles elevados de VEGF-D y PAWP más alta, con respecto a pacientes con menores niveles de VEGF-D en esta cohorte de estudio. Aunque no hay datos disponibles sobre el efecto de los tratamientos dirigidos al VEGF-D en pacientes con CI, HP y HP-EVI, se ha demostrado que un tratamiento temprano con inhibidores de VEGFR3 mejora la obstrucción del lumen y las presiones pulmonares en modelos de HAP basados en Sugen/hipoxia en ratas [76]. Colectivamente, las evidencias disponibles sugieren que el VEGF-D podría ser un biomarcador potencial para distinguir la HP-ICFEr de otras etiologías de HP, pero requiere mayor evaluación en estudios multicéntricos extensos. 
Fig. 3. Ubicación de biomarcadores circulantes potenciales en HP-EVI. BNP, péptido natriurético tipo B/cerebral; ProBNP-NT, proBNP N-terminal; miR-206, microRNA-206; sST2, supresor de tumorigenicidad tipo 2, soluble; H-FABP, proteína de unión a los ácidos grasos, tipo cardiaco; GDF-15, factor de diferenciación de crecimiento; sUPAR, receptor del activador de uroquinasa de

plasminógeno, soluble; MIF, factor inhibidor de la migración de macrófagos; CRP, proteína $\mathrm{C}$ reactiva; ET-1, endotelina 1; ProET-1-CT, pro-ET-1 C-terminal; VEGF-D, factor de crecimiento endotelial vascular D.

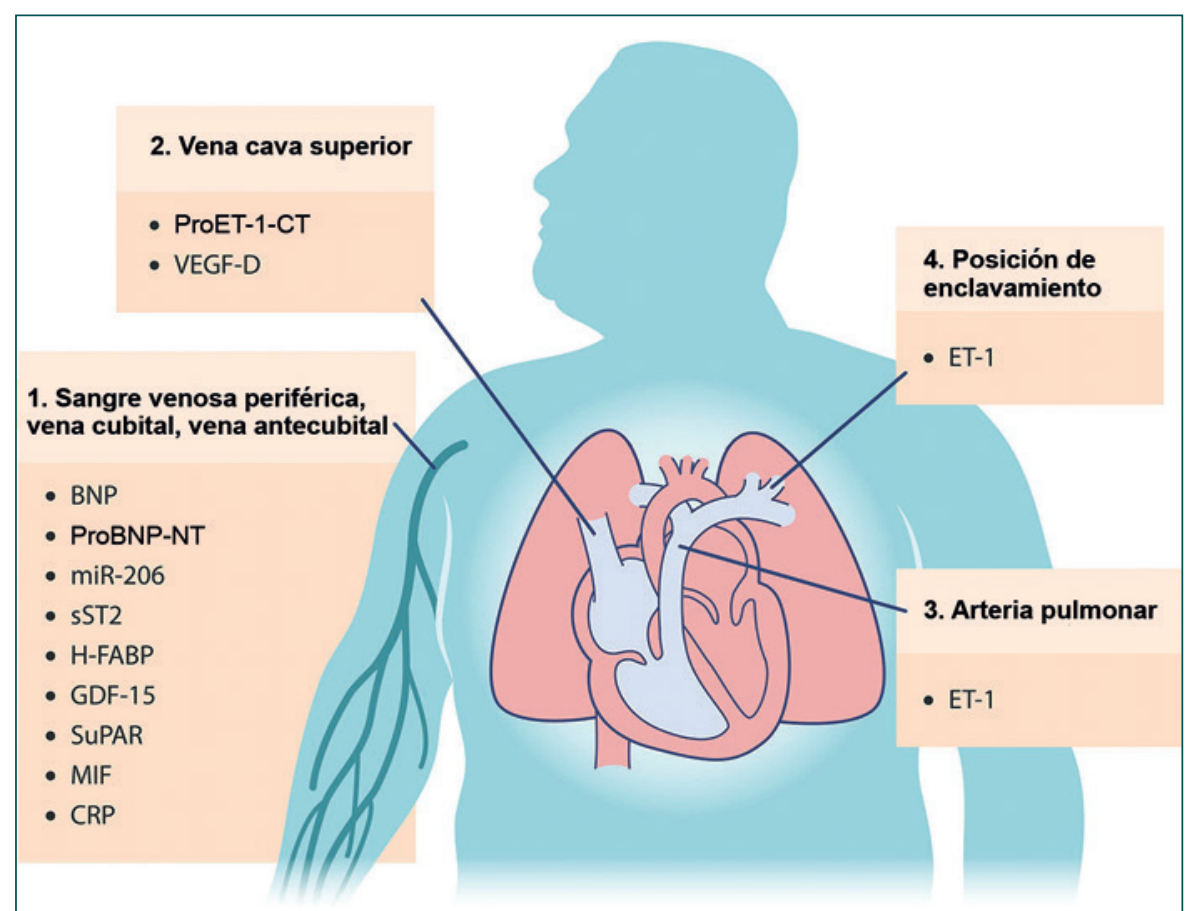

\section{Biomarcadores potenciales: microRNA-206}

Los microRNAs (miRNAs) han despertado gran entusiasmo en la biología molecular en años recientes. Compuestos de RNA no-codificante de cadena única que se autocomplementan en una estructura de asa y bucle, los miRNAs actúan como modificadores postranscripcionales de su RNA mensajero (mRNA), induciendo su degradación o su represión traslacional. Aunque aún existe controversia sobre el mecanismo exacto por el que identifican a sus genes blanco y su papel en la regulación de las enfermedades, los miRNAs se convirtieron en biomarcadores prometedores debido a su estabilidad relativamente alta [77]. Es posible detectarlos extracelularmente, y se les relacionado con una amplia variedad de enfermedades. Varios estudios de caracterización por microarreglos y de arreglos de PCR cuantitativa han reportado miRNAs con utilidad potencial como biomarcadores en IC y HAP (véanse en Fernandez et al., Wong et al. y Boucherat et al. revisiones recientes y detalladas) [22, 78, 79]; sin embargo, sólo se han estudiado unos pocos miRNAs en HP-EVI. Entre ellos, se reportó que los niveles circulantes de miR-204 específico del músculo se encuentran elevados de manera específica en toda la vasculatura pulmonar en pacientes con HAP, pero no en pacientes con HP-EVI [80]. Por otro lado, se encontró que los niveles de miR-206 específico del músculo se hallaban reducidos en el suero de pacientes con HP-ICFEp, HP-ICFEr y HP asociada con enfermedad valvular [42]. De manera notable, la reducción en los niveles de miR-206 en esta cohorte de estudio se correlacionaron con un incremento en los valores de PASP, los niveles de BNP y el diámetro longitudinal de la aurícula izquierda (LAD). Adicionalmente, los autores encontraron que el valor predictivo de miR-206 para detectar HP en EVI mejoró grandemente cuando se combinó con los niveles de BNP y el valor de LAD. Aún se desconoce el papel exacto de miR-206 en la HP-EVI; sin embargo, se demostró que una reducción de miR-206 estabiliza el factor 1 inducible por hipoxia (HIF-1 $\alpha$ ) y el índice de proliferación en PASMCs cultivadas $[81,82]$. En contraste, se encontraron niveles aumentados de miR206 en los pulmones de ratas con HAP inducida por monocrotalina (MCT), y la sobreexpresión cardiaca específica de miR-206 condujo también a hipertrofia cardiaca [83, 84]. Aun cuando la reducción en los niveles circulantes de miR-206 puede compensarse con un incremento en la concentración intracelular de miR206 , se requieren investigaciones adicionales para determinar si el miR-206 circulante puede ser útil en la identificación de HP en pacientes con EVI.

\section{Biomarcadores candidatos a futuro}

Además de los biomarcadores emergentes discutidos anteriormente, varios biomarcadores de distintos procesos en la regulación cardiopulmonar podrían facilitar la caracterización y diferenciación de la HP-EVI o en la determinación del manejo de la enfermedad (Figura 3 y Tabla 2). El supresor de tumorigenicidad tipo 2, soluble (sST2), también conocido como factor similar al receptor de interleucina 1, tipo 1 (IL1RL1), es un ejemplo de tales marcadores. sST2 es un marcador de estrés mecánico y remodelación ventricular, que identifica a pacientes con ICFEr y recientemente se demostró que se encuentra elevado en el plasma de pacientes con HP del grupo 2, con respecto a sujetos ingresados por angiografía coronaria electiva (en quienes se excluyó la CAD) en un estudio retrospectivo en un solo centro realizado por Mirna et al. [85]. Aunque se requiere cautela para interpretar los datos, y hace falta información detallada sobre las características iniciales, 
Tabla 2. Estudios recopilados sobre futuros candidatos de biomarcadores en HP-EVI

\begin{tabular}{|c|c|c|c|c|c|}
\hline Autores (ref. \#) & Biomarcadores & Pacientes $(n)$ & Controles $(n)$ & Hallazgos principales & Correlación con la hemodinamia \\
\hline $\begin{array}{l}\text { Mima et al. } \\
{[85]}\end{array}$ & $\begin{array}{l}\text { sST2, GDF-15, } \\
\text { H-FABP, sUPAR, } \\
\text { BNP }\end{array}$ & $\begin{array}{l}\text { HP grupo } 1(13), \\
\text { HP grupo } 2(35), \\
\text { HP grupo } 3 \text { (7), } \\
\text { HP grupo } 4 \text { (12), } \\
\text { HP grupo } 5 \text { (19), } \\
\text { análisis retrospectivo }\end{array}$ & $\begin{array}{l}\text { Angiografía coronaria } \\
\text { electiva sin CAD (74) }\end{array}$ & $\begin{array}{l}\text { BNP, GDF-15, H-FABP y suPAR están } \\
\text { elevados en pacientes con HP } \\
\text { grupo } 2 \text { y se correlaciona con } \\
\text { algunas mediciones } \\
\text { hemodinámicas }\end{array}$ & 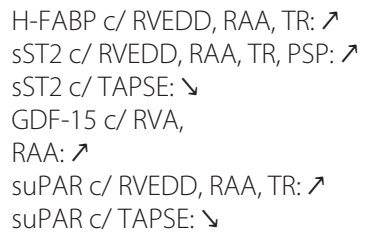 \\
\hline $\begin{array}{l}\text { Luedike et al. } \\
\text { [86] }\end{array}$ & MIF & $\begin{array}{l}\text { ICFEp c/ MIF }>51.58 \\
\text { ng/ml (31), reclutados } \\
\text { prospectivamente }\end{array}$ & $\begin{array}{l}\text { ICFEp c/ MIF } \\
\leq 51.85 \mathrm{ng} / \mathrm{ml}(31)\end{array}$ & $\begin{array}{l}\text { MIF elevada asociada c/ mortalidad/ } \\
\text { hospitalización a los } 180 \text { días y } \\
\text { síntomas de ICFEp más graves }\end{array}$ & MIF c/ PASP: $\nearrow$ \\
\hline $\begin{array}{l}\text { Mazurek et al. } \\
\text { [48] }\end{array}$ & Galectina 3 & $\begin{array}{l}\text { HP-ICFEp (39), } \\
\text { reclutados } \\
\text { prospectivamente }\end{array}$ & $\operatorname{HAP}(37)$ & $\begin{array}{l}\text { Galectina } 3 \text { se correlacionó } \\
\text { positivamente con la mortalidad }\end{array}$ & N/A \\
\hline $\begin{array}{l}\text { DuBrock et al. } \\
\text { [87] }\end{array}$ & $\mathrm{Hs}-\mathrm{CRP}$ & $\begin{array}{l}\text { ICFEp c/ CRP } \\
>3 \text { mg/l (121), análisis } \\
\text { retrospectivo }\end{array}$ & ICFEp c/ CRP $\leq 3 \mathrm{mg} / \mathrm{l}$ & $\begin{array}{l}\text { CRP elevada asociada con mayor } \\
\text { carga de comorbilidad y presencia } \\
\text { de disfunción del VD }\end{array}$ & $\mathrm{N} / \mathrm{A}$ \\
\hline $\begin{array}{l}\text { Nguyen et al. } \\
\text { [88] }\end{array}$ & $\begin{array}{l}\text { Bioenergética } \\
\text { de plaquetas }\end{array}$ & $\begin{array}{l}\text { HP-ICFEp (20), análisis } \\
\text { retrospectivo }\end{array}$ & $\begin{array}{l}\text { Voluntarios } \\
\text { saludables (20) }\end{array}$ & $\begin{array}{l}\text { Las plaquetas de pacientes con } \\
\text { HP-ICFEp muestran elevadas la OCR } \\
\text { máxima y la capacidad respiratoria } \\
\text { de reserva }\end{array}$ & $\begin{array}{l}\text { Capacidad respiratoria de reserva } \\
\text { C/ SWI VD: } \searrow\end{array}$ \\
\hline
\end{tabular}

CAD, enfermedad arterial coronaria; GDF-15, factor de diferenciación de crecimiento 15; H-FABP, proteína de unión a los ácidos grasos, tipo cardiaco; hsCRP, proteína C reactiva de alta sensibilidad; MIF, factor inhibidor de la migración de macrófagos; OCR, tasa de consumo de oxígeno en las plaquetas; RAA, área auricular derecha; RVEDD, diámetro de fin de diástole ventricular derecho; sST2, supresor de tumorigenicidad tipo 2, soluble; suPAR, receptor del activador de uroquinasa de plasminógeno, soluble; SWI, índice de trabajo sistólico; TR, gravedad de regurgitación tricúspide; 7 indica correlación positiva con el biomarcador correspondiente; $\searrow$ indica una correlación inversa con el biomarcador correspondiente.

especialmente de los pacientes del grupo 2, Mirna et al. también mostraron que los pacientes con HP del grupo 2 tuvieron mayores niveles circulantes del factor de diferenciación de crecimiento 15 (GDF-15, un marcador de daño celular e inflamación), de la proteína de unión a los ácidos grasos, tipo cardiaco (H-FABP, un marcador de daño en curso al miocardio) y de la forma soluble del receptor del activador de uroquinasa de plasminógeno (suPAR, un marcador de inflamación en curso) [85]. Adicionalmente, se encontró que los niveles elevados del factor inhibidor de la migración de macrófagos (MIF), una citocina proinflamatoria, se asociaron con mayores valores de PASP y niveles más altos de péptido natriurético en pacientes con ICFEp [86]. Mazurek et al. también mostraron una tendencia hacia niveles más altos de galectina 3 , una proteína de unión con beta-galactósidos que se ha implicado en la inflamación y fibrosis, en el suero recolectado de la arteria pulmonar de pacientes con HP-ICFEp, con respecto a pacientes con HAP [48]. Aunque esto puede estar vinculado con la fibrosis sistémica y la inflamación generalizada que se han observado en poblaciones con ICFEp, en este estudio no se encontraron correlaciones entre los niveles de galectina 3 y cualquier resultado hemodinámico medido en pacientes con HP-ICFEp o HAP [48]. El biomarcador común de inflamación y dificultades cardiovasculares, la proteína $\mathrm{C}$ reactiva (CRP), también ha despertado interés como potencial biomarcador para la HP-EVI. Aplicando un valor de corte de CRP de $>3 \mathrm{mg} / \mathrm{dl}$, el ensayo RELAX reportó que aproximadamente $60 \%$ de los pacientes con ICFEp incluidos tie- nen niveles elevados de CRP [87]. Niveles aumentados de CRP se asociaron con edades menores, valores más altos de IMC, EPOC, fibrilación auricular, disfunción del VD, tolerancia reducida al ejercicio, y mayores niveles circulantes de ET-1, aldosterona y ProBNP-NT. Aunque esto llevó a los autores a concluir que niveles altos de CRP podrían identificar un fenotipo único de pacientes con ICFEp, asociado con inflamación sistémica producto de comorbilidades, los niveles de CRP no se asociaron con HP ni con la función del VI en esta cohorte de estudio [87]. Por último, también se ha demostrado que las plaquetas de pacientes con HP-ICFEp exhiben un fenotipo metabólico distintivo con respecto a las de pacientes con HAP [88]. A diferencia de las plaquetas de pacientes con HAP, sólo se observó un incremento en la capacidad respiratoria máxima, pero no en la tasa glucolítica, en plaquetas de pacientes con HP-ICFEp con respecto a controles saludables. Se encontró que el aumento en la capacidad respiratoria máxima en las plaquetas se asocia con disfunción en el VD, pero no con cambios en mPAP o en la PVR en pacientes con HP-ICFEp [88]. Se requieren estudios adicionales para determinar si la medición de estos factores puede brindar un método para distinguir el fenotipo de pacientes con HP-EVI, identificar a sujetos en riesgo y predecir el desenlace de pacientes con HP-EVI.

Además de los estudios clínicos, los avances recientes en los modelos preclínicos han abierto nuevos caminos en el desarrollo de futuros biomarcadores. Se encontró que los niveles plasmáticos de leptina, una adipocina conocida por inducir la secreción de cito- 
cinas proinflamatorias y la generación de especies reactivas de oxígeno (ROS), están asociados con la gravedad en ratas de la HP-ICFEp inducida por una dieta alta en grasas (DAG) en combinación con el anillado aórtico supracoronario (AAS) y el fármaco antipsicótico olanzapina [89]. También se observaron niveles plasmáticos disminuidos de la adipocina cardioprotectora adiponectina después de la inducción de HP-ICFEp mediada por Sugen en ratas ZSF1, obesas y deficientes del receptor de leptina [90]. De manera interesante, se encontró que la metformina (fármaco de primera línea para la diabetes tipo 2) mejora el síndrome metabólico y las presiones pulmonares en estos modelos al modular la leptina, la adiponectina y otros mecanismos que involucran, al menos en parte, la supresión de la inflamación asociada con la interleucina 6 y la activación de la captación de glucosa en el músculo esquelético mediada por la sirtuina 3 [89, 90]. Más aún, recientemente se demostró que la metformina previene la disfunción del VD al mejorar la resistencia a la insulina y reducir la acumulación de lípidos en el VD en ratones tratados con una DAG [91]. Estos hallazgos llevaron a un ensayo en curso diseñado para evaluar el efecto de la metformina en pacientes con HP-ICFEp (NCT03629340). El nitrito, un precursor dietético del NO, ha mostrado cualidades similares en un modelo de HP-ICFEp en ratas, donde actúa incrementando los niveles de adiponectina y mejorando la resistencia a la insulina en el músculo esquelético [90]. Un estudio reciente de Simon et al. reportó que la inhalación de nitrito redujo la presión pulmonar, la presión en la $\mathrm{AD}$ y la presión capilar de enclavamiento en pacientes con HP-ICFEp [92]. Actualmente está en curso un ensayo clínico para examinar el efecto del nitrito oral en pacientes con HP-ICFEp (NCT03015402). Además, se ha demostrado que las terapias dirigidas a ET-1 y NO con fasudil, un inhibidor de la rho-quinasa (ROCK), también son efectivas en ratas con HP-EVI en etapa terminal inducida por AAS y en pacientes con HP-ICFEp, con resultados más favorables en la población con HPpcC-ICFEp [93, 94].

\section{Resumen y perspectivas a futuro}

Aunque aún existen varios retos a superar, los investigadores en este campo trabajan para expandir la creciente gama de biomarcadores circulantes, que guiarán las futuras investigaciones para facilitar el tamizado, el diagnóstico, el refinamiento de fenotipos específicos y el desarrollo de estrategias terapéuticas. Se requiere consenso en una definición concisa y estandarizada para el fenotipo hemodinámico, junto con datos longitudinales, que consistan en múltiples puntos temporales, y la evaluación en estudios extensos y multicéntricos para validad la viabilidad de los biomarcadores potenciales mencionados anteriormente, y para explorar futuros candidatos. El estudio PVDOMICS, en curso, apoyado por los Institutos Nacionales de Salud y el Instituto Nacional del Corazón, Pulmones y Sangre (NIH/NHLBI) podría asimismo revelar nueva información importante [95]. Puesto que la ICFEp y la HP se reconocen como padecimientos multiorgánicos y sistémicos [96, 97], la búsqueda para identificar biomarcadores extracardiopulmonares significativos podría brindar nuevas pistas sobre este síndrome, clínicamente complejo, y hacer avanzar la investigación sobre HP-EVI.

\section{Contribuciones de los autores}

NT y Y-CL escribieron el artículo.

\section{Financiamiento}

La escritura de esta revisión recibió el apoyo del Instituto Nacional del Corazón, Pulmones y Sangre (R01HL142638) y la Sociedad Norteamericana del Corazón (17SDG33400233).

\section{Conflicto de interés}

Los autores declaran que la investigación se condujo en ausencia de cualquier relación comercial o financiera que pudiera interpretarse como un potencial conflicto de interés.

\section{Agradecimientos}

Agradecemos al Dr. Sergei Snovida por sus útiles comentarios sobre el manuscrito y a Elfy Chiang por su ayuda y por la producción de las figuras.

\section{Información sobre licencias}

Noah Todd, Yen-Chun Lai: Current Understanding of Circulating Biomarkers in Pulmonary Hypertension Due to Left Heart Disease. Front Med (Lausanne). 2020 Oct 7;7:570016 (DOI: 10.3389/fmed.2020.570016). ${ }^{\complement} 2021$ Los Autores (traducción; contribuciones de los autores, abreviadas), protegido por CC BY 4.0 (https://creativecommons.org/licenses/by/4.0/deed.es).

\section{Referencias}

Las referencias están disponibles en www.karger.com/Article/Fulltext/ 000519806. 